\title{
O papel do sotaque nas relações intergrupais: apresentação de uma área de pesquisa
}

\author{
The role of accent in intergroup relations: presentation of a research area
}

\author{
Luana Elayne Cunha de Souza ${ }^{1}$ \\ Leoncio Camino ${ }^{2}$ \\ Tiago Jessé Souza de Lima $^{3}$ \\ Ana Beatriz Gomes Fontenele ${ }^{4}$
}

\begin{abstract}
RESUMO: O presente artigo apresenta uma revisão teórica sobre o estudo do sotaque e sua influência nas relações intergrupais dentro da Psicologia Social. Nesse sentido, analisamos a definição de sotaque e sua centralidade já nos primeiros anos de vida, discutimos sobre o mito de uma língua padrão e apresentamos as principais metodologias no estudo do sotaque. Além disso, analisamos o processo de avaliação dos sotaques, evidenciando a importância que eles têm para a identidade social dos indivíduos, para desencadear o processo de categorização social e formação de estereótipos e, consequentemente, na discriminação em diversos contextos sociais contra falantes de sotaques considerados fora do padrão. Finalmente, analisamos diferentes interpretações acerca da atribuição de valor negativo a falantes de sotaques considerados fora do padrão e propomos que essa avaliação negativa do sotaque funciona como uma justificação aparentemente neutra utilizada por indivíduos preconceituosos para discriminar falantes de sotaques não padrão, ou seja, os membros de grupos minoritários. Deste modo, defendemos que o estigma do sotaque per se não leva à discriminação, isso só vai ocorrer naqueles indivíduos que já tenham uma representação negativa do grupo alvo.
\end{abstract}

Palavras-chave: sotaque; relações intergrupais; preconceito; discriminação; legitimação.

\begin{abstract}
This article presents a theoretical review on the study of the accent and its influence on intergroup relations within Social Psychology. In this sense, we analyze the definition of an accent and its centrality already in the first years of life, we discuss about the myth of a standard language and present the main methodologies in the study of the accent. In addition, we analyze the process of assessment of accents, highlighting the importance its have for the social identity of individuals, to trigger the process of social categorization and stereotyping and, consequently, discrimination in various social contexts against speakers of accents considered non-standard. Finally, we analyze different interpretations of negative evaluations of speakers of accents considered non-standard and propose that this negative assessment of accent works as a seemingly neutral justification used by prejudiced individuals to discriminate non-standard accent speakers, that is, the minority groups. In this way, we argue that the accent stigma per se does not lead to discrimination, this will only occur in those individuals who already have a negative representation of the target group.
\end{abstract}

Keywords: accent; intergroup relations; prejudice; discrimination; legitimacy.

\footnotetext{
1 Professora do Programa de Pós-Graduação em Psicologia da Universidade de Fortaleza - Fortaleza, CE, Brasil. E-mail: luana_elayne@hotmail.com.

2 Professor Emérito da Universidade Federal da Paraíba - João Pessoa, PB, Brasil.

3 Professor da Graduação em Psicologia e do Mestrado Profissional em Administração da Universidade de Fortaleza Fortaleza, CE, Brasil.

${ }^{4}$ Graduanda em Psicologia e Bolsista PIBIC/CNPq da Universidade de Fortaleza - Fortaleza, CE, Brasil.
} 


\section{Introdução}

A linguagem é um poderoso sistema simbólico que oferece um rico repertório de termos lexicais e formas gramaticais onde praticamente cada enunciado reflete uma escolha para o que se fala. Além disso, implica um gradiente de conotativos, significados avaliativos, de tal forma que qualquer pronunciamento transmite algum grau de aceitação ou rejeição, aproximação ou evitação. Não é, portanto, nenhuma surpresa que repertórios discursivos sobre pessoas e grupos podem ter um impacto potencialmente forte nos estereótipos sociais (Fiedler \& Schmid, 2003).

Pessoas facilmente formam impressões de outras pessoas durante a interação social. Além de símbolos visuais, tais como gênero, idade e etnia, a língua desempenha um papel crucial, e muitas vezes um papel primário (Rakić, Steffens, \& Mummendey, 2011) na forma como as pessoas percebem e avaliam umas as outras (e.g., Gallois \& Callan, 1981; Gallois, Callan, \& Johnstone, 1984; Giles \& Marlow, 2011). Além disso, a língua falada é inerentemente variável em todos os níveis, incluindo pronúncia, gramática e vocabulário. Embora partes dessa variabilidade reflitam diferenças idiossincráticas entre os diferentes falantes, uma parcela significativa da mesma é sistemática e reflete as diferenças regionais, sociais e contextuais no uso da linguagem (Lippi-Green, 1997). Como resultado desses padrões estruturados de uso da língua, as formas linguísticas podem se tornar decisivas para as identidades sociais dos falantes (ver Scherer \& Giles, 1979; Silverstein, 2003).

A este respeito, a utilização de determinados idiomas, sotaques (i.e., variedades linguísticas marcadas por uma pronúncia específica) e dialetos (i.e., as variedades linguísticas marcadas por uma gramática específica e vocabulário, para além da pronúncia) podem transmitir uma quantidade significativa de informação social sobre os falantes, tais como a sua distribuição geográfica, etnia e classe social, bem como os estereótipos atribuídos a respeito de seus traços (Callan \& Gallois, 1987; Dragojevic, Berglund, Timothy, \& Blauvelt, 2017; Giles \& Billings, 2004; Fuertes, Gottdiener, Martin, Gilbert, \& Giles, 2012; Gluszek \& Dovidio, 2010a). Ademais, pesquisas sugerem que a maneira como uma pessoa fala tem sido um sinal importante na identificação da pessoa como membro do exogrupo (Edwards, 1999; Giles \& Johnson, 1987; Gluszek \& Dovidio, 2010a; Lippi-Green, 2012). Assim, quando faltam sinais visíveis para distinguir como membros do endogrupo ou do exogrupo, as pessoas usam a linguagem e o sotaque para fazer essa distinção (Kinzler, Shutts, DeJesus, \& Spelke, 2009).

Entretanto, mesmo quando as características visíveis estão presentes, as pessoas, não obstante, se voltam para a linguagem - não para a aparência - para categorizar uma pessoa como pertencendo ao endogrupo ou exogrupo (Hansen, Rakić, \& Steffens, 2014; Rakić et al., 2011). Segundo Kinzler et al. (2009), essa tendência pode derivar do fato de que ao longo da história as pessoas viviam numa tamanha proximidade que fisicamente pareciam bem semelhantes, diferindo muitas vezes em pistas auditivas, seja falando um idioma diferente, dialeto ou com um sotaque diferente. Assim, a única forma de distinguir um amigo de um inimigo potencial era ouvir de perto.

Deste modo, de acordo com o que os pesquisadores têm demonstrado, parece que o sotaque de uma pessoa é uma importante pista social que ativa o processo de categorização social de maneira a classificar o falante como membro do endogrupo ou do exogrupo. De todas as pistas vocais investigadas (por exemplo, tom de voz e velocidade da fala), os sotaques têm recebido, sem dúvida, a maior atenção empírica e são reconhecidos talvez como a marca 
social mais efetiva para o processo de categorização social em diferentes culturas (Fuertes et al., 2012; Keblusek, Giles, \& Maass, 2017).

A partir desta breve apresentação, percebe-se o papel central que o sotaque pode ocupar nas relações intergrupais. De modo a tornar mais claro esse processo, o objetivo principal deste trabalho é apresentar a área de pesquisa sobre o papel que o sotaque desempenha nas relações intergrupais, esclarecendo como ele é utilizado na categorização social, na formação de estereótipos e de impressões acerca dos falantes, e, consequentemente, na discriminação.

De modo mais específico, neste trabalho apresentamos a definição de sotaque, a sua centralidade desde os primeiros anos de vida, a discussão sobre sotaques considerados padrão e não padrão, as principais metodologias de estudo do sotaque, o panorama de pesquisas que demonstram como os sotaques são avaliados e como eles atuam nas relações intergrupais e, finalmente, buscamos apresentar as possíveis explicações para a avaliação negativa que recorrentemente é feita sobre os indivíduos que falam com um sotaque considerado fora do padrão.

\section{Sotaque e sua centralidade desde os primeiros anos de vida}

Sotaque refere-se a um modo de pronúncia (Giles, 1973), que é construído nas interações sociais, na linguagem cotidiana, na cultura popular, e em muitos contextos profissionais. Qualquer que seja o indivíduo, o seu modo de falar e os significados sociais atribuídos a essa característica, juntamente com reações e decisões que derivam dessas atribuições, são questões incontestavelmente significativas no que se refere à comunicação (Giles \& Marlow, 2011).

O fato de as pessoas confiarem tanto na voz e na linguagem, quando se trata de dar sentido aos outros, não deve ser muito surpreendente se considerar que a audição é o primeiro sentido totalmente desenvolvido e utilizado por recém-nascidos (Crystal, 2005; Mehler, Bertoncini, Barrière, \& Jassik-Gerschenfeld, 1978). Na verdade, Nazzi, Jusczyk e Johnson (2000) observaram que as crianças americanas a partir dos cinco meses de idade já são sensíveis a variações dialéticas (ver também, Kinzler, Dupoux, \& Spelke, 2007), parecendo já ter desenvolvido um sistema de som de referência para o Inglês, que as ajuda imediatamente a perceber e reagir se sotaques diferentes são apresentados ao lado de sua língua nativa. Assim, as conexões língua-som feitas durante o processo de aprendizagem da língua nativa continuam a desempenhar um papel central durante toda a vida na comunicação. Isso representa um obstáculo a superar quando se trata do aprendizado de uma segunda língua em um ponto mais tarde na vida, especialmente quando os sotaques são, supostamente, muito mais difíceis de mudar (Lippi-Green, 2012).

De tal modo, com a idade de cinco anos, sotaques podem ser tão potentes nas avaliações sociais das crianças como o sexo ou a cor da pele. Assim, estudos mostram que as crianças preferem amigos do grupo com o mesmo sotaque, independentemente da sua raça (Kinzler et al., 2009) e também atribuem maior confiança para os falantes de línguas nativas (Kinzler, Corriveau, \& Harris, 2011). Além disso, essas preferências não são movidas por uma compreensão prejudicada dos sotaques não nativos, mas sim por uma simples preferência pelo próprio sotaque. Consequentemente, como argumentam Kinzler, Shutts e Correll (2010), 
parece ser que as crianças têm uma tendência natural a usar a linguagem - e até mesmo o sotaque - como um mecanismo social significativo para organizar o mundo social à sua volta.

Ademais, estudos com crianças são importantes para a compreensão de como atitudes linguísticas são aprendidas e desenvolvidas (para um modelo de socialização de atitudes linguísticas, ver Bradac \& Giles, 1991). Em um estudo francês realizado por Girard, Floccia e Goslin (2008), crianças com idade de 5 ou 6 anos eram muito boas em diferenciar discurso nativo de discurso estrangeiro, embora pareciam não notar diferenças dentro da própria língua, ou seja, sotaque francês do norte e do sul. Várias explicações podem ser responsáveis por este fenômeno. Em primeiro lugar, existem muito mais variações entre os diferentes idiomas do que dentro de cada um deles. Segundo, nessa idade, as crianças começam a desenvolver identidades nacionais, portanto, pode ser mais relevante para elas distinguir variedades "nós" versus "eles" do que distinguir formas em sua própria língua. Este efeito diminui com a idade: enquanto o conhecimento aumenta a diferenciação regional, a diferenciação entre nativos e estrangeiros continua a ser altamente persistente ao longo da vida (Giles \& Rakić, 2012).

\section{Sotaques padrão e não padrão: o mito da língua padrão}

Durante a história do seu desenvolvimento, linguagens normalmente passam por um processo chamado de padronização. O que isto significa é que, de muitas variedades diferentes da mesma língua (por exemplo, diferentes dialetos), uma forma única de definir a gramática, ortografia e pronúncia é estabelecida como a única correta (Dragojevic, Giles, \& Watson, 2013). Ademais, cabe salientar que a escolha pela forma a ser considerada padrão é justamente aquela que representa a forma de falar dos membros do grupo majoritário. Neste sentido, os indivíduos que representam minorias sociais são considerados como tendo um sotaque fora do padrão.

A noção de língua padrão parece ser muito natural nas mentes das pessoas, embora, na verdade, constitua uma construção social, sendo essa falácia muitas vezes referida como o mito de uma língua padrão (Lippi-Green, 2012). Geralmente, as variedades de linguagem padrão possibilitam às pessoas acesso a fóruns políticos, econômicos e educacionais e oportunidades, enquanto os falantes de variedades de linguagem não padrão são confrontados com estigmatização (Gluszek \& Dovidio, 2010a). Um estudo desenvolvido na Holanda exemplifica o quanto o fato de ter um sotaque padrão proporciona uma avaliação geral muito mais positiva em diferentes aspectos do que as diferentes variedades regionais de sotaque holandês (Grondelaers, van Hout, \& Steegs, 2010). Além disso, um estudo desenvolvido nos EUA mostra que falantes de sotaques não nativos relatam sentimentos de pertença mais baixos com os EUA, e esse efeito pode ser mediado por problemas percebidos na comunicação (Gluszek \& Dovidio, 2010b). Ou seja, embora se trate da mesma língua, as pessoas tendem a perceber os sotaques dos indivíduos considerados fora do padrão como difíceis de compreender.

A noção de língua ou sotaque padrão é bastante problemática, mas mais ainda é a noção "falar sem sotaque" ou "ausência de sotaque". Em muitos exemplos da vida cotidiana, falantes com sotaque não padrão são aqueles acusados de terem "um sotaque". Consequentemente, no Brasil, por exemplo, os atores das novelas passam por um tratamento com um fonoaudiólogo para tentarem "perder seu sotaque" (Medeiros, 2006). Nos Estados Unidos, um número crescente de cursos e workshops estão sendo oferecidos com a promessa de 
ajudar os falantes do sotaque não padrão a perderem seu sotaque. Enquanto à primeira vista não parece ter nada de muito errado, na verdade essa noção é completamente equivocada e só reforça o mito de que existe uma língua padrão que deve ser adotada por todos. Ademais, cabe reforçar que todos os indivíduos têm um sotaque, isso faz parte da sua identidade.

\section{Principais metodologias no estudo do sotaque}

Um número razoável de técnicas tem sido adotado para estudar as atitudes linguísticas relacionadas aos sotaques, tais como a identificação direta de qual o sotaque em questão e o uso de mapas que expressam uma série de variedades linguísticas com diferentes significados sociais (ver Preston, 1993; Ryan, Giles \& Hewstone, 1988). Entretanto, a mais popular delas é a técnica do disfarce combinado (Machted-Guise Technique, MGT) desenvolvida por Lambert, Hodgson, Gardner e Fillenbaum (1960), e desde então adotada em muitas culturas.

Os experimentos com a MGT apresentam aos ouvintes passagens ou expressões vocais lidas por pessoas bilíngues ou que falam mais de um dialeto, as quais conseguem adotar vários disfarces de variedades linguísticas específicas. Este procedimento tem a vantagem do controle experimental, uma vez que variáveis interferentes (e.g., investimento prosódico, paralinguístico e emocional) são supostamente mantidas constantes através dos disfarces evocados. Em outras palavras, e embora não esteja isenta de críticas (ver Grondelaers et al., 2010), a técnica assegura que diferenças entre os disfarces são baseadas em características da variedade da linguagem per se, ao invés de ser devido a diferenças vocais que frequentemente existem entre os falantes (Fuertes et al., 2012).

Contudo, esta técnica pode não ser a mais adequada em diferentes contextos, uma vez que ela foi desenvolvida para sociedades bilíngues. Em contextos em que essa realidade não se aplica, muito raramente uma pessoa conseguirá falar mais de uma língua e, principalmente, mais de um sotaque de forma autêntica. Neste sentido, outra técnica utilizada pode ser a "Verbal Guise Technique" (VGT), a qual utiliza indivíduos diferentes para cada condição do sotaque. Embora esta técnica seja criticada pela potencial falta de controle sobre as variações em características paralinguísticas (e.g., tom de voz) entre os diferentes falantes, ela tem a importante vantagem de empregar sotaques naturais ao invés de sotaques falsos, os quais podem representar apenas estereótipos dos falantes (Edwards, 1982).

Deste modo, pesquisas a serem desenvolvidas no Brasil para analisar o preconceito linguístico contra diversas variedades regionais de sotaque podem vir a utilizar a técnica VGT, uma vez que as diferenças entre os sotaques brasileiros são bem demarcadas e, claramente, um indivíduo que nasceu no sul do país apresentará uma variedade do português brasileiro bem diferente de um indivíduo que nasceu no nordeste do país. Ademais, mesmo dentro de uma mesma região do Brasil as diferenças entre os sotaques podem ser bem demarcadas e facilmente reconhecidas por qualquer indivíduo, a exemplo das diferenças entre o sotaque de um cearense e um paraibano.

\section{Avaliação dos sotaques e sua centralidade para a compreensão das relações intergrupais}

No que concerne à avaliação que os indivíduos têm sobre o sotaque "não padrão", em traços de atratividade social e benevolência, como simpatia, falantes do sotaque não padrão 
têm conseguido melhores avaliações, ainda que nem sempre de forma consistente (por exemplo, Dailey, Giles, \& Jansma, 2005; para uma meta-análise, ver Fuertes et al., 2012). De fato, o valor atribuído ao sotaque britânico (estrangeiro) nos EUA e, em contrapartida, o sotaque americano na Austrália são casos ilustrativos desse ponto (Stewart, Ryan, \& Giles, 1985; Bayard, Weatherall, Gallois, \& Pittam, 2001). Neste sentido, quando a identidade endogrupal e lealdade são salientes, sotaques fora do padrão podem ter prestígio encoberto para alguns de seus falantes (Marlow \& Giles, 2008; Ryan, 1979). Este efeito está em consonância com o modelo de conteúdos estereotípicos de Fiske, Cuddy, Glick e Xu (2002), que mostram como os estereótipos atribuídos a grupos estigmatizados (e.g., pessoas idosas) compensam a falta de competência atribuída por atribuições elevadas de simpatia (warmth). No entanto, trabalhos nesta tradição tem, até esta data, como foco a classificação dos rótulos conceituais de diferentes grupos sociais (por exemplo, Fiske et al., 2002), e não características vocais deles. A questão que surge (ver Ryan, 1979) é por que variedades de linguagem fora do padrão persistem mesmo sendo estigmatizadas? Além do poder da aprendizagem nos primeiros anos de vida, uma explicação plausível para essa pergunta é fornecida pelo papel que a língua desempenha para a identidade social.

As pessoas são cognitivamente complexas e têm, simultaneamente, (ou são percebidas como tendo) diferentes identidades sociais para recorrer, sendo as identidades sociais (positivas) cruciais para o seu bem-estar. A teoria da identidade social (TIS; Tajfel \& Turner, 1979) aborda questões de identidade e fornece uma estrutura teórica frutífera para compreender atitudes linguísticas, tanto com relação ao falante quanto ao ouvinte. A teoria da identidade etnolinguística (ELIT, ver Giles \& Johnson, 1981, 1987) - uma teoria orientada para a linguagem baseada na TIS e na teoria de acomodação da comunicação - ressalta o vínculo linguagem-identidade. Segundo este modelo, a língua desempenha funções psicológicas centrais de reforço da identidade social positiva de um falante, especialmente com relação à etnia. Portanto, não é de surpreender que uma variedade de língua utilizada pelas pessoas é um sinal muito forte na determinação de quem é um membro do endogrupo ou do exogrupo (Yzerbyt, Leyens, \& Bellour, 1995). Em relação a este último exemplo, participantes mostraram um efeito de exclusão forte (ou seja, mais prontamente excluíram os falantes com um sotaque não claramente identificável) quando lhe pediram para identificar outros membros do endogrupo. Consequentemente, os encontros podem ser considerados mais em termos intergrupais quando as duas partes não reconhecem uns aos outros como indivíduos, ou seja, como membros de (diferentes) grupos sociais (ver Cargile \& Giles, 1997).

Assim, enquanto poderá ser objetivamente difícil parar de falar com um sotaque não padrão (ou não nativo), de um ponto de vista sociopsicológico isso pode ser realmente contraproducente até mesmo para tentar, porque o sotaque constitui uma parte fundamental da identidade social de um indivíduo e da sua herança cultural. Na verdade, identidades sociais positivas são essenciais para assegurar autoestima positiva e bem-estar (Tajfel, 1981). Como previamente estabelecido, falantes de sotaque não padrão (especialmente imigrantes) ao longo do tempo podem se sentir menos pertencentes à sociedade de acolhimento e, como resultado, terem uma experiência de alienação e empobrecido da imagem endogrupal. No entanto, isto poderia ser mediado e atenuado pela identificação com o próprio grupo de falantes do seu sotaque não padrão. Isto foi apoiado por um grupo de afro-americanos que relataram estar sendo alvo de discriminação, o que influenciou negativamente o seu bemestar, mas, ao mesmo tempo, promoveu uma identificação mais forte com o endogrupo que, 
por sua vez, ajudou a restaurar um bem-estar positivo (Branscombe, Schmitt, \& Harvey, 1999; ver também Wright \& Bougie, 2007).

A percepção negativa dos estereótipos frente à fala com um idioma diferente ou um sotaque não padrão envolve vários aspectos. Indivíduos que têm um sotaque não nativo ou regional são vistos como menos inteligentes e competentes do que pessoas com o sotaque padrão, e como falando uma linguagem pobre (para uma revisão, ver Gluszek \& Dovidio, 2010a). Pesquisas têm identificado três dimensões nas quais as avaliações tendem a convergir: 1) status social que inclui avaliações sobre a inteligência, competência, ambição, educação e classe social do falante; 2) solidariedade que inclui avaliações sobre a similaridade do falante com o ouvinte, atratividade, benevolência e confiança e 3) dinamismo que referese ao nível de atividade e vivacidade do falante (Fuertes, et al., 2012; Giles \& Billings, 2004; Mulac, Hanley, \& Prigge, 1974; Zahn \& Hopper, 1985).

Falantes com sotaque não padrão tendem a ser classificados como tendo baixo status, especialmente quando seus sotaques são percebidos como difíceis de compreender (Bresnahan, Ohashi, Nebashi, Liu, \& Shearman, 2002). Embora tais falantes sejam algumas vezes percebidos com escores mais altos na dimensão de solidariedade. Uma meta-análise concluiu que, em geral, falantes do sotaque não padrão são avaliados mais negativamente do que os falantes do sotaque padrão em todas as três dimensões: status, solidariedade e dinamismo (Fuertes et al., 2012).

Isso evidencia que a sensibilidade para outras origens linguísticas tem consequências reais para os falantes e ouvintes igualmente. As atitudes das pessoas para aqueles que falam de maneira diferente tendem a ser negativas (Gluszek \& Dovidio, 2010a) e resultam em estereótipos, preconceitos e discriminação em todos os aspectos da vida cotidiana, incluindo, por exemplo, a escola, o mercado de trabalho e os meios de comunicação (Lippi-Green, 2012).

De maior importância, o sotaque não somente torna acessível a categorização endogrupo-exogrupo, como também permite aos indivíduos avaliarem a extensão na qual a "qualidade avaliada do sotaque" se ajusta à maneira de falar mais desejável socialmente. Neste sentido, a discriminação baseada na percepção do sotaque difere da discriminação étnica ou racial (Goto, Gee, \& Takeuchi, 2002), de forma que a maneira como uma pessoa fala é usada como base para avaliações arbitrárias e, ao contrário de outras formas de discriminação, é comumente aceita e percebida como legítima na sociedade (Gluszek \& Dovidio, 2010a). Como aponta Lippi-Green (2012), indivíduos não sentem remorso em discriminar com base na língua, porque eles agem como se o sotaque fosse um critério decisivo para a exclusão, uma desculpa para se afastar e recusar reconhecer o outro.

Deste modo, parece razoável pensar que a percepção dos indivíduos sobre a "qualidade do sotaque" do alvo pode funcionar como uma razão justificável para o comportamento discriminatório contra este alvo, de maneira que os indivíduos veem seu comportamento como correto e legítimo (Souza, Pereira, Camino, Lima, \& Torres, 2016). A seguir, serão discutidas três áreas - escola, emprego e mídia - onde falar com um idioma diferente ou com um sotaque "não padrão" tem consequências sociais importantes para os indivíduos.

\section{Escola}

O mito da linguagem ou sotaque padrão (Dragojevic et al., 2013; Lippi-Green, 2012) está profundamente enraizado nos sistemas educacionais na maioria dos países das Américas 
(Hornberger, 2000; Lippi-Green, 2012). Desde cedo, as crianças aprendem a ler e a escrever de acordo com a imposição de uma forma apropriada de escrita e idioma - a que já é considerada como o padrão para dado idioma (Gluszek \& Hansen, 2013). Mesmo nos países que apoiam abertamente a educação bilíngue e multilíngue como o Canadá, uma única linguagem é a que muitas vezes proporciona mais prestígio social (Bourhis, Montaruli, \& Amiot, 2007).

Embora haja uma aparente aceitação das variedades linguísticas existentes em cada país, isso rapidamente muda para a recomendação de que todos os alunos deveriam aprender "a língua de comunicação mais ampla". A maneira como o professor ensina a gramática aos estudantes, afirmando ser esta a correta, motiva o chamado bullying linguístico (Souza, 2012). Este tipo de preconceito, geralmente é sustentado pelos meios de comunicação em massa, pela gramática normativa e pelos livros didáticos.

Assim, uma das principais razões pelas quais as variedades não padrão de linguagem e pronúncia são desencorajadas nos sistemas educativos nas Américas é que as crianças que crescem falando de maneira diferente enfrentam uma série de desvantagens quando entram na idade adulta jovem, dos preconceitos do sistema de ensino superior para a discriminação no local de trabalho (Gluszek \& Hansen, 2013).

\section{Emprego}

Atitudes negativas em relação à fala não padrão vão além dos muros das instituições de ensino e afetam falantes que entram em um emprego e prosseguem em suas carreiras. Discriminação no emprego com base na língua ou sotaque é prevalente em países do continente americano. Nos Estados Unidos, por exemplo, pesquisas revelaram que as pessoas que falam com sotaques regionais ou não nativos são percebidas como menos contratáveis (por exemplo, Hosoda e Stone-Romero, 2010; Huang, Frideger, \& Pearce, 2013; Segrest Purkiss, Perrewé, Gillespie, Mayes, \& Ferris, 2006) e, quando contratadas, têm maior probabilidade de serem atribuídas a posições de baixo status do que aquelas pessoas com sotaque padrão (de la Zerda \& Hopper, 1979).

Além disso, pesquisadores têm demonstrado que a língua (ou línguas) que uma pessoa fala tem consequências sobre o emprego e a renda. Nos EUA, funcionários mexicanosamericanos falando com um sotaque mexicano ganham menos do que os trabalhadores mexicanos-americanos com um sotaque americano, independente da linguagem de proficiência (Davila, Bohara, \& Saenz, 1993). No Canadá, resultados semelhantes foram relatados (Creese \& Kambere, 2003; Hakak, Holzinger, \& Zikic, 2010).

É importante notar que a maioria dos países das Américas proíbem a discriminação baseada na língua e no sotaque e muitos dos países latino-americanos garantem uma educação bilíngue para indígenas (UNESCO, 2003). No entanto, mesmo em países com fortes leis antidiscriminação, como os EUA, a lei permite exceções. Por exemplo, os empregadores não podem contratar ou demitir uma pessoa se eles acreditam que o seu "sotaque interfere seriamente no seu desempenho de trabalho" (US Equal Employment Opportunity Commission, 2012). No entanto, os critérios objetivos para avaliar os efeitos do sotaque no desempenho de uma pessoa são inexistentes, e os tribunais muitas vezes dependem de empregadores ou de suas próprias opiniões subjetivas para determinar se o sotaque de uma pessoa é problemático (Lippi-Green, 1994). 
Além disso, os empregadores nos Estados Unidos prontamente admitem que discriminam com base na aparência externa de uma pessoa ou no seu sotaque (Lippi-Green, 1994). Nesse sentido, aqueles que percebem o seu sotaque como uma barreira em interações sociais e profissionais podem experimentar resultados negativos para a sua saúde mental. Por exemplo, Wated e Sanchez (2006) observaram que a posse de um sotaque não nativo serve como um preditor significativo do estresse experimentado no ambiente de trabalho entre trabalhadores hispânicos nos EUA.

Em uma pesquisa realizada em 26 dos 27 países da União Europeia, 34\% dos respondentes de uma amostra representativa acreditam que o sotaque de um candidato para uma vaga de trabalho o colocaria em desvantagem comparado com um candidato igualmente qualificado "sem sotaque". Entre os gerentes, que frequentemente estão na posição de contratar, este número sobe para 45\% (Comissão Europeia, 2008). Neste sentido, tendo em conta que o preconceito pode afetar o comportamento e as decisões de maneira tanto consciente quanto inconsciente (Devine, 1989; Dovidio, 2001), permitindo que os empregadores decidam subjetivamente se "ter um sotaque" constitui uma deficiência comunicativa, esses preconceitos são capazes de afetar as vidas de falantes de sotaques não nativos sem quaisquer salvaguardas (Lippi-Green, 2012).

\section{Mídia}

O mito de uma linguagem ou sotaque padrão e concepções estereotipadas associadas com formas não padrão também são perpetuadas pela mídia. Nos Estados Unidos, uma análise de conteúdo de uma amostra aleatória de desenhos animados de TV a cabo para crianças revelou que os vilões falam com sotaques estereotipados como negativos (alemão, russo, e outros do Leste Europeu), enquanto os heróis falam com sotaques do inglês americano e britânico (Dobrow \& Gidney, 1998). Padrões similares de estereótipos aparecem em filmes e programas de TV dirigidos a adultos (Lippi-Green, 2012). Por exemplo, nas novelas da televisão brasileira, os personagens com papéis subalternos (por exemplo, empregados e pessoas de classe baixa) falam com sotaques característicos da região Nordeste enquanto que os atores principais geralmente falam com o sotaque dito "padrão". Uma pesquisa realizada com os próprios comunicadores de mídia do Nordeste do Brasil mostrou que esses profissionais suavizam características do falar nordestino durante o exercício profissional para se adequar ao padrão preconizado pelo mercado de trabalho (Ramos, Lira, \& Roazzi, 2015).

Embora os filmes costumem usar o sotaque como "atalho mental" para facilmente categorizar e retratar personagens de forma estereotipada, ao mesmo tempo, eles exigem atores que tenham um "sotaque flexível". Assim, estúdios de cinema e departamentos universitários de teatro oferecem uma variedade de cursos de formação de sotaque para ensinar como adquirir a capacidade de falar com outros sotaques e como neutralizar seus próprios.

Neste sentido, os meios de comunicação não só promovem a variedade padrão, mas também moldam o que é considerado padrão. Por exemplo, o maior canal da TV brasileira promove um "Português padrão", que é na verdade outra variedade da língua inexistente, ou seja, ensinada artificialmente por fonoaudiólogos aos atores e apresentadores para eliminar as características naturais dos seus sotaques (Massini-Cagliari, 2004). Ademais, forte influência da mídia foi demonstrada em um estudo de atitudes em relação a diferentes sotaques do Português falado em cinco grandes cidades brasileiras. Nenhum dos sotaques foi 
percebido como apropriado para se tornar o padrão para todo o país (Ramos, 1997). Além disso, os falantes dos sotaques considerados mais adequados para se tornar um padrão, se percebiam falando de forma mais semelhante à linguagem utilizada nos meios de comunicação, demonstrando que a mídia desempenha um papel importante na definição das normas de pronúncia no Brasil (Medeiros, 2006; Ramos, 1997).

Sotaques naturais fora do padrão aparecem nos meios de comunicação em pequena escala, mas os meios de comunicação em larga escala são mais prováveis de retratar atores imitando vários sotaques (em graus variados de sucesso). Este fenômeno pode servir como base para uma crença errônea entre os falantes com pronúncia não padrão de que os sotaques podem ser facilmente alterados ou mesmo eliminados (Lippi-Green, 2012; Scovel, 2000). Embora a força do sotaque diminua à medida que uma pessoa resida em um determinado país e aumente o uso da língua não nativa, os pesquisadores em geral, tendem a concordar que as pessoas que aprendem uma língua mais tarde na vida são altamente improváveis de adquirir uma pronúncia semelhante à nativa do lugar (por exemplo, Flege, 1988; Piske, MacKay, \& Flege, 2001; Scovel, 2000). Mesmo os atores que são bem-sucedidos em imitar outros sotaques colocam um esforço considerável em ter que falar de uma forma que não é natural para eles e voltam a sua forma habitual de falar quando as câmeras estão desligadas (Lippi-Green, 2012).

Assim, as maneiras pelas quais os meios de comunicação retratam sotaques e linguagens podem afetar significativamente a forma como as crianças e os adultos respondem às pessoas que falam de uma forma não padrão. Especificamente, as pessoas passam uma parte significativa da sua vida assistindo televisão. Esta, por sua vez, afeta a visão das pessoas sobre a realidade e também seus comportamentos (Bryant \& Oliver, 2009), incluindo as suas percepções sobre a fala, favorecendo o uso de variantes gramaticais padrões (PEUL, 1991).

\section{Possíveis explicações para a avaliação negativa que falantes com sotaques fora do padrão}

Diferentes interpretações têm sido propostas para explicar porque falantes com sotaque não padrão são avaliados menos favoravelmente do que falantes do sotaque padrão. Algumas dessas interpretações, um tanto provocativas, têm argumentado que falantes não nativos são rebaixados não tanto por causa do preconceito social ou por estereótipos préexistentes, mas sim devido ao aumento da carga cognitiva experienciada pelos ouvintes a fim de entenderem as mensagens com sotaque não padrão (Dragojevic \& Giles, 2016; Lev-Ari \& Keysar, 2010; Pearson \& Dovidio, 2014). Enquanto este argumento pode ser relevante em tarefas onde uma pessoa tem que discriminar rapidamente uma breve declaração ambígua como verdade ou não, é menos provável que o mesmo se aplique em encontros da vida social, onde outras informações sobre o falante podem estar presentes e quando amostras de fala são mais longas, oferecendo assim mais tempo para se acostumar a essa variedade de linguagem.

Dovidio e Gluszek (2012) também consideram que a discriminação de falantes fora do padrão é resultado do uso de sotaque (não padrão) e comportamento não verbal que causam disfluência e aumentam vieses intergrupais nos ouvintes. Para estes estudiosos, parece que o sotaque (não padrão) e o comportamento não verbal facilitam o viés intergrupal porque aumentam a força dos limites intergrupais e desta forma contribuem para interações disfluentes. Mesmo que este modelo reconheça parcialmente a percepção enviesada dos 
outros, ao mesmo tempo, ele se baseia na noção de que há algo essencialmente disfluente nos falantes não padrão que causam menor compreensão. Contudo, essa visão é bastante problemática por ao menos duas razões. Primeiro, pesquisas sugerem que embora sotaques desconhecidos possam inicialmente interromper o processo de fluência, a adaptação e normalização seguem rapidamente e os participantes demonstram uma completa recuperação na velocidade de processamento (Floccia, Goslin, Girard, \& Konopczynski, 2006). Segundo, não somente se assume que um ouvinte é como um receptor objetivo de uma mensagem, mas também negligencia outros aspectos que são conhecidos por influenciar as percepções das pessoas, como as identidades sociais e as emoções despertadas.

Curiosamente, é esta ideia do "incompreensível" que é um argumento central da ideologia da linguagem padrão; a justificação e racionalização de porque falantes não padrão (e não nativos) são de alguma forma "inferiores". Não obstante, segundo Souza et al. (2016), essas justificações ou racionalizações de que um determinado sotaque é incompreensível ou fora do padrão podem atuar como formas de legitimar a discriminação contra o membro do exogrupo (o falante não padrão), não pelo seu sotaque em si, mas sim pelo preconceito que o indivíduo carrega sobre o grupo do qual o falante faz parte (grupo minoritário). Deste modo, a avaliação aparentemente neutra que os indivíduos fazem do sotaque não padrão pode ser usada para justificar a discriminação contra membros de grupos minoritários, de maneira a legitimar esse comportamento e não o vê-lo como discriminatório.

Neste sentido, de acordo com Souza et al. (2016), a discriminação de falantes de sotaques não padrão pode ser explicada de acordo com as relações intergrupais existentes entre os grupos, e não devido a uma mera disfluência na comunicação. Ademais, a discriminação com base no sotaque do alvo ocorrerá somente quando a categoria social eliciada por este sotaque tiver uma conotação negativa para o observador (Hansen \& Dovidio, 2016). Em outras palavras, a mera categorização endogrupo ou exogrupo ativada pelo sotaque não é suficiente para levar à discriminação, esta só ocorrerá quando a categoria social for alvo de preconceito já existente para com o grupo do alvo. Deste modo, a discriminação com base no sotaque do alvo só ocorre em indivíduos preconceituosos (Souza, 2014).

\section{Considerações finais}

Neste trabalho, discutimos acerca do conceito do sotaque e de como este pode ajudar na compreensão das dinâmicas intergrupais desde os primeiros anos de vida dos indivíduos, salientando que a maneira como um indivíduo fala pode transmitir uma quantidade significativa de informação social sobre ele, como sua distribuição geográfica, etnia e classe social. Nesse sentido, a literatura mostra que, mesmo quando as características visíveis estão presentes nas relações sociais, as pessoas utilizam a linguagem para a identificação de outrem como membro do endogrupo ou exogrupo. Além disso, a linguagem passa por um processo de padronização, sendo definida, dentre variedades da mesma língua, uma maneira "mais correta" de se falar. Salientamos que esse processo de escolha de uma língua ou variedade linguística padrão é, na realidade, um mito, pois todas as variedades representam as características culturais de um determinado grupo social. Não obstante, esse mito da língua padrão é reforçado constantemente nas relações intergrupais e pode ser facilmente percebido em diferentes contextos sociais, a exemplo da escola, mercado de trabalho e mídia.

Ademais, constatamos que, além do sotaque desencadear o processo de categorização social em endogrupo e exogrupo, ele também é utilizado para a formação de estereótipos e 
para a discriminação, sendo os falantes do sotaque não padrão avaliados menos favoravelmente do que falantes do sotaque padrão. Dessa forma, vimos que muitas interpretações existem para explicar essa avaliação negativa do sotaque não padrão. Lev-Ari e Keysar (2010) argumentam que ocorre um aumento da carga cognitiva experienciada pelos ouvintes para entender a fala com sotaque não padrão. Entretanto, esse argumento reforça a ideia do "incompreensível", sendo uma justificativa e racionalização do motivo de falantes não padrão serem "inferiores". Outra interpretação considera que há uma disfluência nos falantes não padrão, o que causa uma menor compreensão nos ouvintes (Dovidio \& Gluszek, 2012; Dragojevic et al., 2017). A este respeito, Souza et al. (2016) defendem que essas justificativas e racionalizações da incompreensibilidade de um sotaque parecem ser uma maneira de legitimar a discriminação contra o falante não padrão (membro do exogrupo), não pelo sotaque em si mas pela posição grupal que ele ocupa na sociedade. Assim, esses autores argumentam que a discriminação com base no sotaque do alvo só ocorrerá quando a categoria social já for alvo de preconceito para o grupo alvo, não sendo a mera categorização em endogrupo e exogrupo suficiente para levar à discriminação.

Por fim, espera-se que este trabalho contribua para um melhor entendimento acerca da centralidade que o sotaque tem nas relações intergrupais, de modo a salientar novos aspectos que estão relacionados ao modo como os indivíduos se relacionam e discriminam membros de grupos minoritários. Ademais, espera-se também que esta revisão teórica possa promover um maior interesse dos psicólogos sociais brasileiros sobre essa área de investigação. A este respeito, uma agenda de pesquisa pode vir a ser desenvolvida de modo a primeiramente conhecer quais as variedades linguísticas brasileiras que são desvalorizadas socialmente e se os falantes dessas variedades fora do padrão são estigmatizados e discriminados em função do seu modo de falar.

\section{Referências}

Bayard, D., Weatherall, A., Gallois, C., \& Pittam, J. (2001). Pax Americana? Accent attitudinal evaluations in New Zealand, Australia, and America. Journal of Sociolinguistics, 5, 22-49.

Bourhis, R. Y., Montaruli, E., \& Amiot, C. E. (2007). Language planning and French-English bilingual communication: Montreal field studies from 1977 to 1997. International Journal of the Sociology of Language, 185, 187-224.

Bradac, J. J., \& Giles, H. (1991). Social and educational consequences of language attitudes. Moderna Språk, 85, 1-11.

Branscombe, N. R., Schmitt, M. T., \& Harvey, R. D. (1999). Perceiving pervasive discrimination among African Americans: Implications for group identification and well-being. Journal of Personality and Social Psychology, 77, 135-149.

Bresnahan, M. J., Ohashi, R., Nebashi, R., Liu, W. Y., \& Shearman, S. M. (2002). Attitudinal and affective response toward accented English. Language and Communication, 22, 171-185.

Bryant, J., \& Oliver, M. B. (2009). Media effects: advances in theory and research (3a Ed.). New York: Routledge.

Callan, V. J., \& Gallois, C. (1987). Anglo-Australians' and immigrants' attitudes toward language and accent: A review of experimental and survey research. International Migration Review, 21, 48-69.

Cargile, A. C., \& Giles, H. (1997). Understanding language attitudes: Exploring listener affect and identity. Language and Communication, 17, 195-217.

Creese, G., \& Kambere, E. N. (2003). What colour is your English? Canadian Review of Sociology and Anthropology, 40, 565-573. 
Crystal, D. (2005). The Cambridge encyclopedia of language. In D. Crystal (Org.). The Cambridge encyclopedia of language (pp. 229-259). Cambridge, UK: Cambridge University Press.

Dailey, R. M., Giles, H., \& Jansma, L. L. (2005). Language attitudes in an Anglo-Hispanic context: The role of the linguistic landscape. Language and Communication, 25, 27-38.

Davila, A., Bohara, A. K., \& Saenz, R. (1993). Accent penalties and the earnings of Mexican Americans. Social Science Quarterly, 74, 902-916.

De la Zerda, N., \& Hopper, R. (1979). Employment interviewers' reactions to Mexican American speech. Communication Monographs, 46, 126-134.

Devine, P. G. (1989). Stereotypes and prejudice: Their automatic and controlled components. Journal of Personality and Social Psychology, 56, 5-18.

Dobrow, J. R., \& Gidney, C. L. (1998). The good, the bad, and the foreign: The use of dialect in children's animated television. The Annals of the American Academy of Political and Social Science, 557, 105-119.

Dovidio, J. F. (2001). On the nature of contemporary prejudice: The third wave. Journal of Social Issues, 57, 829849.

Dovidio, J. F., \& Gluszek, A. (2012). Accents, nonverbal behavior, and intergroup bias. Em H. Giles (Org.), The handbook of intergroup communication (pp. 87-99). New York: Routledge.

Dragojevic, M., \& Giles, H. (2016). I don't like you because you're hard to understand: The role of processing fluency in the language attitudes process. Human Communication Research, 42, 396-420.

Dragojevic, M., Berglund, C., \& Blauvelt, T. (2017). Figuring out who's who: The role of social categorization in the language attitudes process. Journal of Language and Social Psychology, 1, 1-23.

Dragojevic, M., Giles, H., \& Watson, B. M. (2013). Language ideologies and attitudes: A dynamic foundational framework. In H. Giles \& B. M. Watson (Eds.), The social meanings of languages, dialects, and accents: An international perspective. New York: Peter Lang.

Edwards, J. (1982). Language attitudes and their implications among English speakers. In E. B. Ryan \& H. Giles (Eds.). Attitudes toward language variation: Social and applied contexts (pp. 20-33). London: Edward Arnold.

Edwards, J. (1999). Refining our understanding of language attitudes. Journal of Language and Social Psychology, 18, 101-110.

Fiedler, K., \& Schmid, J. (2003). How language contributes to persistence of stereotypes as well as other, more general, Intergroup Issues. In R. Brown \& S. L. Gaertner (Eds.). Blackwell Handbook of Social Psychology: Intergroup processes (pp. 261-280). Blackwel Publishing.

Fiske, S. T., Cuddy, A. J. C., Glick, P., \& Xu, J. (2002). A model of (often mixed) stereotype content: Competence and warmth respectively follow from perceived status and competition. Journal of Personality and Social Psychology, 82, 878-902.

Flege, J. E. (1988). Factors affecting degree of perceived foreign accent in English sentences. Journal of the Acoustical Society of America, 84, 70-79.

Floccia, C., Goslin, J., Girard, F. d. r., \& Konopczynski, G. (2006). Does a regional accent perturb speech processing? Journal of Experimental Psychology: Human Perception and Performance, 32, 1276-1293.

Fuertes, J. N., Gottdiener, W., Martin, H., Gilbert, T. C., \& Giles, H. (2012). A meta-analysis of the effects of speakers' accents on interpersonal evaluations. European Journal of Social Psychology, 42, 120-133.

Gallois, C., \& Callan, V. J. (1981). Personality judgments elicited by accented English speech. Journal of CrossCultural Psychology, 12, 347-359.

Gallois, C., Callan, V. J., \& Johnstone, M. (1984). Personality judgments of Australian and Aborigine and White speakers: Ethnicity, sex, and context. Journal of Language and Social Psychology, 3, 39-57.

Giles, H. (1973). Communicative effectiveness as a function of accented speech. Speech Monographs, 40, 330331.

Giles, H., \& Billings, A. (2004). Language attitudes. In A. Davies \& E. Elder (Eds.). Handbook of applied linguistics (pp. 187-209). Oxford, UK: Blackwell 
Giles, H., \& Johnson, P. (1981). The role of language in ethnic group relations. In J. C. Turner \& H. Giles (Eds.). Intergroup behavior (pp. 199-243). Oxford, UK: Blackwell.

Giles, H., \& Johnson, P. (1987). Ethnolinguistic identity theory: A social psychological approach to language maintenance. International Journal of the Sociology of Language, 68, 69-99.

Giles, H., \& Marlow, M. (2011). Theorizing language attitudes: Past frameworks, an integrative model, and new directions. In C. Salmon (Ed.). Communication yearbook 35 (pp. 161-197). Thousand Oaks: Sage.

Giles, H., \& Rakić, T. (2012). Language attitudes: The social determinants and consequences of language variation. In T. Holtgraves (Ed.). The Oxford handbook of language and social psychology. New York: Oxford University Press.

Girard, F., Floccia, C., \& Goslin, J. (2008). Perception and awareness of accents in young children. British Journal of Developmental Psychology, 26, 409-433.

Gluszek, A., \& Dovidio, J. F. (2010a). A social psychological perspective on the stigma of non-native accents in communication. Personality and Social Psychology Review, 14, 214-237.

Gluszek, A., \& Dovidio, J. F. (2010b). Speaking with a nonnative accent: Perceptions of bias, communication, and belonging. Journal of Language and Social Psychology, 29, 224-234.

Gluszek, A., \& Hansen, K. (2013). Language attitudes and The Americas. In H. Giles \& B. M. Watson (Eds.). The social meanings of languages, dialects and accents: An international perspective. New York: Peter Lang.

Goto, S. G., Gee, G. C., \& Takeuchi, D. T. (2002). Strangers still? The experience of discrimination among Chinese Americans. Journal of Community Psychology, 30, 211-224.

Grondelaers, S., van Hout, R., \& Steegs, M. (2010). Evaluating regional accent variation in Standard Dutch. Journal of Language and Social Psychology, 29, 101-116.

Hakak, L. T., Holzinger, I., \& Zikic, J. (2010). Barriers and paths to success: Latin American MBAs' views of employment in Canada. Journal of Managerial Psychology, 25, 159-176.

Hansen, K., \& Dovidio, J. F. (2016). Social dominance orientation, nonnative accents, and hiring recommendations. Cultural Diversity and Ethnic Minority Psychology, 22(4), 544-551.

Hansen, K., Rakić, T., \& Steffens, M. C. (2012). When actions speak louder than words: Preventing discrimination of nonstandard speakers. Journal of Language and Social Psychology, 33(1), 68-77.

Hornberger, N. H. (2000). Bilingual education policy and practice in the Andes-Peru, Ecuador, and Bolivia: Ideological paradox and intercultural possibility. Anthropology and Education Quarterly, 31, 173-201.

Hosoda, M., \& Stone-Romero, E. (2010). The effects of foreign accents on employment-related decisions. Journal of Managerial Psychology, 25, 113-132.

Huang, L., Frideger, M., \& Pearce, J. L. (2013). Political skill: Explaining the effects of nonnative accent on managerial hiring and entrepreneurial investment decisions. Journal of Applied Psychology, 98, 10051017.

Keblusek, L., Giles, H., \& Maass, A. (2017). Communication and group life: How language and symbols shape intergroup relations. Group Processes \& Intergroup Relations, 20(5), 632-643.

Kinzler, K. D., Corriveau, K. H., \& Harris, P. L. (2011). Children's selective trust in native-accented speakers. Developmental Science, 14, 106-111.

Kinzler, K. D., Dupoux, E., \& Spelke, E. S. (2007). The native language of social cognition. Proceedings of the National Academy of Science, 104, 12577-12580

Kinzler, K. D., Shutts, K., \& Correll, J. (2010). Priorities in social categories. European Journal of Social Psychology, 40, 581-592.

Kinzler, K. D., Shutts, K., DeJesus, J., \& Spelke, E. S. (2009). Accent trumps race in guiding children's social preferences. Social Cognition, 27, 623-634.

Lambert, W. E., Hodgson, E. R., Gardner, R. C., \& Fillenbaum, S. (1960). Evaluational reactions to spoken languages. The Journal of Abnormal and Social Psychology, 60, 44-51. 
Lev-Ari, S., \& Keysar, B. (2010). Why don't we believe non-native speakers? The influence of accent on credibility. Journal of Experimental Social Psychology, 46, 1093-1096.

Lippi-Green, R. (1994). Accent, standard language ideology, and discriminatory pretext in the courts. Language in Society, 23, 163-198.

Lippi-Green, R. (1997). English with an accent. London: Routledge.

Lippi-Green, R. (2012). English with an accent: Language, ideology, and discrimination in the United States (2nd ed.). New York: Routledge.

Marlow, M. L., \& Giles, H. (2008). Who You Tink You, Talkin Propah? Hawaiian Pidgin Demarginalised. Journal of Multicultural Discourses, 3, 53-68.

Massini-Cagliari, G. (2004). Language policy in Brazil: Monolingualism and linguistic prejudice. Language Policy, 3, 3-23.

Medeiros, A. L. (2006). Sotaques na TV. São Paulo, Brazil: Annablume

Mehler, J., Bertoncini, J., Barrière, M., \& Jassik-Gerschenfeld, D. (1978). Infant recognition of mother's voice. Perception, 7, 491-497

Mulac, A., Hanley, T. D., \& Prigge, D. Y. (1974). Effects of phonological speech foreignness upon three dimensions of attitude of selected American listeners. The Quarterly Journal of Speech, 60, 411-420.

Nazzi, T., Jusczyk, P. W., \& Johnson, E. K. (2000). Language discrimination by English-learning 5-month-olds: Effects of rhythm and familiarity. Journal of Memory and Language, 43, 1-19.

Pearson, A. R., \& Dovidio, J. F. (2014). Intergroup fluency: How processing experiences shape intergroup cognition and communication. In J. P. Forgas, O. Vincze \& J. Laszlo (Eds.), Social cognition and communication (pp. 101-120). New York, NY: Psychology Press.

PEUL - Research Group on the Use of Language (1991). Results of an integrated sociolinguistic study. International Journal of the Sociology of Language, 89, 25-46.

Piske, T., MacKay, I. R. A., \& Flege, J. E. (2001). Factors affecting degree of foreign accent in an L2: A review. Journal of Phonetics, 29, 191-215.

Preston, D. R. (1993). Folk dialectology. In D. R. Preston (Ed.). American dialect research (pp. 333-378). Philadelphia, PA: John Benjamins.

Rakić, T., Steffens, M. C., \& Mummendey, A. (2011). Blinded by the accent! The minor role of looks in ethnic categorization. Journal of Personality and Social Psychology, 100, 16-29.

Ramos, J. M. (1997). Avaliação de dialetos brasileiros, o sotaque. Revista de Estudos da Linguagem, 5, $103-125$.

Ramos, L. M., Lira, Z. S., \& Roazzi, A. (2015). Sotaque e telejornalismo: representações de comunicadores de mídia nordestinos. Revista CEFAC, 17(6), 1987-1999.

Ryan, E. B. (1979). Why no low-prestige language varieties persist? In H. Giles \& R. N. St. Clair (Eds.). Language and social psychology (pp. 145-157). Oxford, UK: Blackwell.

Ryan, E. B., Giles, H., \& Hewstone, M. (1988). The measurement of language attitudes. In U. Ammon, N. Dittmar, \& K. J. Mattheier (Eds.). Sociolinguistics: An international handbook of the science of language (Vol. II, pp. 1068-1081). Berlin: Mouton de Gruyter.

Scherer, K. R., \& Giles, H. (Eds.). (1979). Social markers in speech. Cambridge, UK: Cambridge University Press.

Scovel, T. (2000). A critical review of the critical period research. Annual Review of Applied Linguistics, 20, 213223.

Segrest Purkiss, S. L., Perrewé, P. L., Gillespie, T. L., Mayes, B. T., \& Ferris, G. R. (2006). Implicit sources of bias in employment interview judgments and decisions. Organizational Behavior and Human Decision Processes, 101, 152-167.

Silverstein, M. (2003). Indexical order and the dialectics of sociolinguistic life. Language \& Communication, 23, 193-229. 
Souza, C. V. C. (2012). Bullying linguístico: uma nova visão sobre o preconceito e a discriminação. Trabalho de conclusão de curso de Pós-graduação Lato Sensu em Revisão de texto, Centro Universitário de Brasília, Brasília, Brasil.

Souza, L. E. C. (2014). A influência da categorização pelo sotaque na discriminação. Tese de Doutorado não publicada, Programa de Pós-graduação em Psicologia Social, Universidade Federal da Paraíba, Paraíba, Brasil.

Souza, L. E. C., Pereira, C. R., Camino, L., Lima, T. J. S., \& Torres, A. R. R. (2016). The legitimizing role of accent on discrimination against immigrants. European Journal of Social Psychology, 46, 609-620.

Stewart, M. A., Ryan, E. B., \& Giles, H. (1985). Accent and social class effects on status and solidarity evaluations. Personality \& Social Psychology Bulletin, 11, 98-105.

Tajfel, H. (1981). Human groups and social categories. Cambridge: University Press.

Tajfel, H., \& Turner, J. C. (1979). An integrative theory of intergroup conflict. In W. G. Austin \& S. Worchel (Eds.). The social psychology of intergroup relations (pp. 33-47). Monterey, CA: Brooks-Cole.

UNESCO. (2003). Linguistic rights in national constitutions. Retirado de http://www.unesco.org/most/In2nat.htm\#With.

US Equal Employment Opportunity Commission. (2012). National origin discrimination. Retirado de http://www.eeoc.gov/laws/types/nationalorigin.cfm.

Wated, G., \& Sanchez, J. I. (2006). The role of accent as a work stressor on attitudinal and health-related work outcomes. International Journal of Stress Management, 13, 329-350.

Wright, S. C., \& Bougie, È. (2007). Intergroup Contact and Minority-Language Education. Journal of Language and Social Psychology, 26, 157-181.

Yzerbyt, V., Leyens, J.-P., \& Bellour, F. (1995). The ingroup overexclusion effect: Identity concerns in decisions about group membership. European Journal of Social Psychology, 25, 1-16.

Zahn, C. J., \& Hopper, R. (1985). Measuring language attitudes: The speech evaluation instrument. Journal of Language and Social Psychology, 4, 113-123. 